\title{
The Response to Oxidative Damage Correlates with Driver Mutations and Clinical Outcome in Patients with Myelofibrosis
}

\author{
Elena Genovese ${ }^{1}$, Margherita Mirabile ${ }^{1}$, Sebastiano Rontauroli ${ }^{1}$, Stefano Sartini ${ }^{1}$, Sebastian Fantini ${ }^{1}$, \\ Lara Tavernari $^{1}$, Monica Maccaferri ${ }^{2}$, Paola Guglielmelli ${ }^{3} \mathbb{D}$, Elisa Bianchi ${ }^{1} \mathbb{D}$, Sandra Parenti ${ }^{1}$, \\ Chiara Carretta ${ }^{1}{ }_{(\mathbb{D}}$, Selene Mallia ${ }^{1}$, Sara Castellano ${ }^{4,5,6}{ }^{(\mathbb{D}}$, Corrado Colasante ${ }^{5} \mathbb{D}$, Manjola Balliu ${ }^{3}$, \\ Niccolò Bartalucci ${ }^{3}$, Raffaele Palmieri ${ }^{7}$ (D), Tiziana Ottone ${ }^{7,8}$, Barbara Mora ${ }^{9}$, Leonardo Potenza ${ }^{5}$, \\ Francesco Passamonti ${ }^{9}$, Maria Teresa Voso ${ }^{7,8} \mathbb{D}$, Mario Luppi ${ }^{5}$, Alessandro Maria Vannucchi ${ }^{3}(\mathbb{D}$, \\ Enrico Tagliafico ${ }^{4,5,+} \mathbb{D}$, Rossella Manfredini ${ }^{1, *,+} \mathbb{D}$ \\ and on behalf of the Mynerva (MYeloid NEoplasms Research Venture AIRC) $\ddagger$
}

Citation: Genovese, E.; Mirabile, M.; Rontauroli, S.; Sartini, S.; Fantini, S.;

Tavernari, L.; Maccaferri, M.;

Guglielmelli, P.; Bianchi, E.;

Parenti, S.; et al. The Response to Oxidative Damage Correlates with

Driver Mutations and Clinical

Outcome in Patients with

Myelofibrosis. Antioxidants 2022, 11,

113. https://doi.org/10.3390/

antiox11010113

Academic Editor: Stefania Filosa

Received: 3 December 2021

Accepted: 1 January 2022

Published: 5 January 2022

Publisher's Note: MDPI stays neutral with regard to jurisdictional claims in published maps and institutional affiliations.

Copyright: (C) 2022 by the authors. Licensee MDPI, Basel, Switzerland. This article is an open access article distributed under the terms and conditions of the Creative Commons Attribution (CC BY) license (https:// creativecommons.org/licenses/by/ $4.0 /)$.
1 Centre for Regenerative Medicine, Life Sciences Department, University of Modena and Reggio Emilia, 41125 Modena, Italy; elena.genovese@unimore.it (E.G.); margherita.mirabile@unimore.it (M.M.); sebastiano.rontauroli@unimore.it (S.R.); stefano.sartini@unimore.it (S.S.); sebastian.fantini@unimore.it (S.F.); lara.tavernari@unimore.it (L.T.); elisa.bianchi@unimore.it (E.B.); sandra.parenti@unimore.it (S.P.); chiara.carretta@unimore.it (C.C.); selene.mallia@unimore.it (S.M.)

2 Department of Laboratory Medicine and Pathology, Diagnostic Hematology and Clinical Genomics, AUSL/AOU Policlinico, 41124 Modena, Italy; maccaferri.monica@policlinico.mo.it

3 Center of Research and Innovation of Myeloproliferative Neoplasms (CRIMM), Department of Experimental and Clinical Medicine, University of Florence, Careggi University Hospital, 50134 Florence, Italy; paola.guglielmelli@unifi.it (P.G.); manjola.balliu@unifi.it (M.B.); niccolo.bartalucci@unifi.it (N.B.); a.vannucchi@unifi.it (A.M.V.)

4 Center for Genome Research, University of Modena and Reggio Emilia, 41125 Modena, Italy; sara.castellano@unimore.it (S.C.); enrico.tagliafico@unimore.it (E.T.)

5 Department of Medical and Surgical Sciences, University of Modena and Reggio Emilia, AUSL/AOU Policlinico, 41124 Modena, Italy; 248358@studenti.unimore.it (C.C.); leonardo.potenza@unimore.it (L.P.); mario.luppi@unimore.it (M.L.)

6 PhD Program in Clinical and Experimental Medicine, University of Modena and Reggio Emilia, 41124 Modena, Italy

7 Department of Biomedicine and Prevention, University of Tor Vergata, 00133 Rome, Italy; raffaele.f.palmieri@gmail.com (R.P.); tiziana.ottone@uniroma2.it (T.O.); voso@med.uniroma2.it (M.T.V.)

8 Santa Lucia Foundation, I.R.C.C.S., Neuro-Oncohematology, 00179 Rome, Italy

9 Division of Hematology, Ospedale ASST Sette Laghi, University of Insubria, 21110 Varese, Italy; barbara.mora@asst-settelaghi.it (B.M.); francesco.passamonti@asst-settelaghi.it (F.P.)

* Correspondence: rossella.manfredini@unimore.it

$+\quad$ These authors contributed equally to this work.

$\ddagger$ Membership of the Group is provided in the https://progettomynerva.it/ (accessed on 1 December 2021)

Abstract: Myelofibrosis (MF) is the Philadelphia-negative myeloproliferative neoplasm characterized by the worst prognosis and no response to conventional therapy. Driver mutations in JAK2 and CALR impact on JAK-STAT pathway activation but also on the production of reactive oxygen species (ROS). ROS play a pivotal role in inflammation-induced oxidative damage to cellular components including DNA, therefore leading to greater genomic instability and promoting cell transformation. In order to unveil the role of driver mutations in oxidative stress, we assessed ROS levels in CD34+ hematopoietic stem/progenitor cells of MF patients. Our results demonstrated that ROS production in CD34+ cells from CALR-mutated MF patients is far greater compared with patients harboring JAK2 mutation, and this leads to increased oxidative DNA damage. Moreover, CALR-mutant cells show less superoxide dismutase (SOD) antioxidant activity than JAK2-mutated ones. Here, we show that high plasma levels of total antioxidant capacity (TAC) correlate with detrimental clinical features, such as high levels of lactate dehydrogenase (LDH) and circulating CD34+ cells. Moreover, in JAK2mutated patients, high plasma level of TAC is also associated with a poor overall survival (OS), and multivariate analysis demonstrated that high TAC classification is an independent prognostic factor allowing the identification of patients with inferior OS in both DIPSS lowest and highest categories. 
Altogether, our data suggest that a different capability to respond to oxidative stress can be one of the mechanisms underlying disease progression of myelofibrosis.

Keywords: myelofibrosis; calreticulin; janus kinase 2; oxidative stress; reactive oxygen species; superoxide dismutase; 8-hydroxy-2'-deoxy-guanosine; total antioxidant capacity

\section{Introduction}

The maintenance of physiological balance between pro-oxidant and antioxidant factors is crucial for proper cellular functions, and the loss of this equilibrium causes oxidative stress. Oxidative stress in turn induces the pathologic accumulation of reactive oxygen species (ROS) that exert their damaging effect on membrane lipids, proteins and DNA [1]. For these reasons, oxidative stress has been investigated in a broad variety of tumors and hematological malignancies, including myeloproliferative neoplasms (MPNs) [2,3].

MPNs are clonal hematopoietic stem cells disorders characterized by excessive production of terminally differentiated myeloid cells [4]. MPNs include three main entities: polycythemia vera (PV), essential thrombocythemia (ET) and primary myelofibrosis (PMF). In particular, PMF patients show high serum level of pro-inflammatory cytokines accompanied by a high level of intracellular ROS, which play a crucial role in chronic inflammation and genomic instability [5]. Moreover, PV and ET can evolve into secondary myelofibrosis (SMF) giving rise to post-PV myelofibrosis (PPV-MF) and post-ET myelofibrosis (PET-MF) [6].

The molecular pathogenesis of PMF has been widely studied in the last years. JAK2, $C A L R$ and MPL variants are considered driver mutations since their acquisition plays a key role in leading the malignant clonal expansion [7]. Moreover, these mutations are associated with other DNA alterations which contribute to the onset of the inflammatory state characterized by an excessive production of ROS [2,8]. Several findings support the pathogenetic model in which the oxidative stress contributes to the chronic inflammation and genomic instability [9]. According to this model, an excess of ROS produced by the malignant clone triggers a vicious self-perpetuating circle, in which ROS activate proinflammatory pathways that in turn create more ROS [10,11].

The JAK2V617F mutation results in a ligand-independent activation of JAK2 kinase and downstream phosphorylation of STATs [12,13]. It has been demonstrated that JAK2 mutation leads to an increase of ROS levels through various mechanisms, including $\mathrm{p} 47 \mathrm{phox}$ phosphorylation [14] and constitutive activation of AKT/mTOR signaling pathway [15]. Conversely, the inhibition of ROS production could prevent the development of MPNs in a $J A K 2 V 617 F$ knock-in mouse model, thus confirming the involvement of oxidative stress in the pathogenesis of these myeloid malignancies [16].

In 2013, two independent groups reported the discovery of mutations in CALR gene in $60-80 \%$ of $J A K 2$ and MPL unmuted ET and PMF patients $[17,18]$. CALR is a major chaperone in the endoplasmic reticulum (ER) playing multiple roles in several cellular processes, such as quality control of protein folding and calcium homeostasis [19]. Mutations in CALR gene mainly consist of insertions or deletions that induce +1 -base pair frameshift resulting in a partial loss of the C-terminal domain. It has been shown that physical interaction of mutant CALR homomultimers with MPL causes its constitutive activation [20-22]. Wild-type CALR is involved in oxidative stress response in different cell types [23]. Moreover, CALR overexpression has been shown to increase cell sensitivity to $\mathrm{H}_{2} \mathrm{O}_{2}$-induced cytotoxicity [24], indicating that CALR plays a critical role in oxidative stress-induced apoptosis.

We recently demonstrated that $\mathrm{K} 562$ cells overexpressing mutated CALR display increased sensitivity to oxidative stress, leading to augmented oxidative DNA damage [25]. In order to confirm our previous results in primary cells, here we have assessed oxidative stress levels in CD34+ hematopoietic progenitor cells (HSPCs) from myelofibrosis (MF) patients. In the present study, we describe how JAK2 and CALR mutations impact on 
oxidative stress response in CD34+ cells. Finally, we measured total antioxidant capacity (TAC), an analyte frequently used to assess the antioxidant status of biological samples, in plasma of $J A K 2$ and $C A L R$-mutated patients.

\section{Materials and Methods}

\subsection{Patients and Samples}

This study was conducted using Human CD34+ Hematopoietic Stem/Progenitor Cells (HSPCs) and plasma samples. Human CD34+ cells were purified from peripheral blood (PB) of 17 healthy donor (HD) and from 34 patients with a diagnosis of primary $(n=18)$ and secondary $(n=16)$ myelofibrosis $(\mathrm{MF}), 20$ of them harboring JAK2 mutations (i.e., JAK2V617F or JAK2 exon 12 mutations) and 14 of them harboring CALR mutations (i.e., type 1/type 1-like or type 2/type 2-like). Plasma samples were collected from $129 \mathrm{MF}$ patients ( $n=86 J A K 2, n=43 C A L R)$. All subjects, recruited from 4 Italian centers, provided informed written consent. MF was diagnosed according to 2016 World Health Organization criteria [26]. The study was conducted in accordance with the Declaration of Helsinki and was approved by local ethics committees.

\subsection{Plasma Isolation}

Blood samples were collected via venipuncture in EDTA-containing tubes. Plasma was separated as already described [27]. Hemolyzed samples were identified by spectrophotometric analyses, measuring the absorbance of hemoglobin at $414 \mathrm{~nm}$ using the NanoDrop ND-1000 spectrophotometer (Thermo Fisher Scientific, Waltham, MA, USA). As reported in literature [28], samples with a hemoglobin absorbance $>0.3$ were considered hemolyzed and were excluded from the study. Plasma was then aliquoted and stored at $-80^{\circ} \mathrm{C}$.

\subsection{Human CD34+ Hematopoietic Stem/Progenitor Cells Purification}

Human CD34+ cells were collected from peripheral blood (PB) using a Ficoll-Hypaque density gradient. Briefly, blood sample was diluted 1:4 with Phosphate-Buffered Saline (PBS) with $2 \mathrm{mM}$ EDTA and centrifuged at $800 \times g$ for $20 \mathrm{~min}$ at room temperature (RT) in a swinging-bucket rotor without brake. Following centrifugation, the mononuclear cell (MNC) ring was then recovered and washed twice with PBS. CD34+ were isolated through the immunomagnetic CD34+ positive selection using CD34 MicroBead Kit UltraPure (Miltenyi Biotec, Bergish Gladbach, Germany).

\subsection{CD34+ Cells Culture Conditions}

CD34+ cells were seeded in 24-well plates at $5 \times 10^{5}$ cells $/ \mathrm{mL}$ in Iscove's modified Dulbecco's medium (IMDM) (EuroClone, Pero, Milan, Italy) containing 10\% Human Serum (Sigma-Aldrich, St. Louis, MO, USA), SCF (50 ng/mL), FLT3-ligand (50 ng/mL), TPO (20 ng/mL), IL-6 (10 ng/mL) and IL-3 (10 ng/mL) (all from Miltenyi Biotec, Bergish Gladbach, Germany), as previously described [29]. To induce oxidative stress, CD34+ cells were treated with Melittin, the main constituent and principal toxin of bee venom with powerful oxidant activity [30]. Briefly, CD34+ cells were seeded at $1 \times 10^{6}$ cells $/ \mathrm{mL}$ in IMDM medium supplemented with 10\% Human Serum and Melittin $5 \mu \mathrm{g} / \mathrm{mL}$ (SigmaAldrich, St. Louis, MO, USA) for 6 and $24 \mathrm{~h}$ at $37^{\circ} \mathrm{C}$ in a humidified atmosphere with $5 \% \mathrm{CO}_{2}$.

\subsection{Detection of Intracellular ROS Levels}

The redox-sensitive fluorochrome 5-(and 6)-chloromethyl-2', $7^{\prime}$-dichlorodihydroflurescein diacetate dye (CM-H2DCFDA) (Invitrogen, Waltham, MA, USA) was used to measure the intracellular ROS. Briefly, CD34+ cells were treated with Melittin $5 \mu \mathrm{g} / \mathrm{mL}$ and intracellular ROS concentration was evaluated at 6 and $24 \mathrm{~h}$. In total, $5 \times 10^{4}$ treated/untreated CD34+ cells were loaded with $2 \mu \mathrm{M}$ CM-H2DCFDA for $20 \mathrm{~min}$ at $37^{\circ} \mathrm{C}$. Before flow cytometry analysis, the cells were removed from loading buffer and incubated in growth medium for $30 \mathrm{~min}$ at $37^{\circ} \mathrm{C}$. Data acquisition and analysis were performed using a BD FACSCanto II 
(Becton Dickinson, BD, Franklin Lakes, NJ, USA). At least 100,000 events were detected for each sample to guarantee the statistical significance. Data were analyzed by FlowJo version 10.8 (Becton Dickinson, BD, Franklin Lakes, NJ, USA).

\subsection{Annexin V/PI Staining}

Apoptosis was evaluated by Annexin V assay (Annexin V-FITC Kit, Trevigen Inc., Minneapolis, MN, USA) following manufacturer protocol. Briefly, $5 \times 10^{5} \mathrm{CD} 34+$ cells from 5 JAK2 and 5 CALR-mutated samples were washed with cold PBS and incubated in $100 \mu \mathrm{L}$ Annexin $\mathrm{V}$ incubation reagent for $15 \mathrm{~min}$ at room temperature in the dark. After staining, cells were analyzed by using a BD FACSCanto II (BD Biosciences; San Jose, CA, USA). At least 10,000 events were counted for each sample to ensure statistical relevance.

\subsection{Measurement of SOD Activity}

SOD activity was measured by means of Superoxide Dismutase (SOD) Colorimetric Activity Kit (Invitrogen, Waltham, MA, USA) following manufacturer instruction. Briefly, CD34+ cells were treated for $24 \mathrm{~h}$ with Melittin $5 \mu \mathrm{g} / \mathrm{mL}$. Then, $1 \times 10^{6}$ CD34+ cells have been lysed in $250 \mu \mathrm{L}$ of cold PBS with Halt ${ }^{\mathrm{TM}}$ Protease and Phosphatase Inhibitor (Thermo Fisher Scientific, Waltham, MA, USA) through 3 cycles of $10 \mathrm{~s}$ (Amplitude: 50\%) using Branson digital sonicator model 450 (Branson, Brookfield, CT, USA). The protein concentration was determined by using Bradford Reagent (Sigma-Aldrich, St. Louis, MO, USA) and SOD activity were normalized based on total amount of protein were used to perform the assay.

\subsection{Measurement of 8-OHdG Levels}

8-hydroxy-2'-deoxy-guanosine (8-OHdG) is one of the major DNA oxidative modifications that can be generated by hydroxylation of the deoxyguanosine residues. Levels of 8-OHdG can be detected by enzyme-linked immunosorbent assay (ELISA). To this end, genomic DNA was extracted from CD34+ cells after treatment with Melittin $5 \mu \mathrm{g} / \mathrm{mL}$ for $24 \mathrm{~h}$ by means of DNeasy Blood and Tissue kit (Qiagen, Hilden, Germany). The same amount of genomic DNA of CD34+ cells $(1.5 \mu \mathrm{g})$ was resuspended in $100 \mu \mathrm{L}$ water and used for the detection of 8-OHdG level by means of the OxiSelectTM Oxidative DNA Damage ELISA Kit (Cell Biolabs, San Diego, CA, USA), following the manufacture's instruction.

\subsection{Measurement of TAC Levels}

Total antioxidant capacity was measured by means of the Total Antioxidant Capacity Assay Kit (Colorimetric) (ABCAM, Cambridge, UK), following the manufacturer's instruction. Briefly, plasma samples have been diluted 1:50 in PBS and was added an equal amount of protein mask, reagent supplied by the kit that allows the analysis of the small antioxidant molecules. The assay is based on antioxidant-based conversion of $\mathrm{Cu}^{2+}$ ion into $\mathrm{Cu}^{+}$, and detection of reduced $\mathrm{Cu}^{+}$ion chelated with a colorimetric probe at $570 \mathrm{~nm}$. Standard curve was prepared by using Trolox, a water-soluble tocopherol analogue, and antioxidant capacity was quantified as molar Trolox equivalents.

\subsection{Measurement of L-Lactate Levels}

Levels of plasmatic L-Lactate were measured by means of Lactate-Glo ${ }^{\mathrm{TM}}$ Assay (Promega, Madison, WI, USA) following manufacturer's instructions. Plasma samples from $43 \mathrm{MF}$ patients were diluted 1:100 in PBS. This assay couples the detection of lactate oxidation and NADH production with a bioluminescent NADH detection system. The luminescent signal is proportional to the amount of lactate in the sample and increases until all lactate is consumed, at which point a stable luminescent signal is achieved.

\subsection{Statistical Analysis}

Data were analyzed with GraphPad Prism8 version 8.4.0 (Graph Pad Software, San Diego, CA, USA); $p$ value $<0.05$ was considered significant. Comparisons between healthy 
donors and patients were reported with median with 95\% CI (confidence interval) and analyzed with Mann-Whitney U test. Comparisons between treated and not treated cells were reported with median with 95\% CI and analyzed with Wilcoxon matched-pairs signed rank test. The comparison between $6 \mathrm{~h}$ and $24 \mathrm{~h}$ treated cells was reported with a histogram with median with $95 \% \mathrm{CI}$ and analyzed with Wilcoxon matched-pairs signed rank test. Correlation with categorical variables was assessed using Mann-Whitney U test or, for multiple comparisons, using Kruskal-Wallis test, while correlation with continuous variables was tested using simple linear regression. To compare the distribution of a variable in patients' groups, the Chi-square test was used. All the clinical parameters were evaluated at the time of samples collection. Overall survival (OS) was calculated from the date of sample collection to the date of last follow-up or death occurrence; OS analyses were performed with the Kaplan-Meier method and the log-rank test was used to compare the curves. Multivariate analyses for OS were carried out by means of Cox proportional hazard regression and Wald test was used to compute $p$ value using $R$ version 3.4.1 (R Core Team 2021, Vienna, Austria).

\section{Results}

3.1. Intracellular ROS Quantification and Apoptosis Evaluation in CD34+ Cells from JAK2 or CALR-Mutated MF Patients

Intracellular ROS levels were assessed by flow cytometry in CD34+ cells from 20 JAK2and $14 C A L R$-mutated MF patients compared with $17 \mathrm{HDs}$ (Figure S1), before and after the induction of oxidative stress by Melittin treatment as previously described [30].

Our data show that a statistically significant increase of ROS is measurable in MF untreated cells compared to HD cells (Figure 1a,b). Nevertheless, $6 \mathrm{~h}$ treatment with Melittin induces ROS accumulation in both MF and HD CD34+ cells (Figure 1b). Regarding $J A K 2$ - and CALR-mutated cells, Figure 1c shows that ROS production in CD34+ cells from CALR-mutated patients is significantly higher than in JAK2-mutated cells. Moreover, after Melittin treatment $C A L R$-mutated cells show significantly higher ROS levels than $J A K 2$-mutated ones (Figure 1c).

These differences are more remarkable after $24 \mathrm{~h}$. CALR-mutated CD34+ cells are almost completely unable to reduce intracellular ROS level, while JAK2-mutated CD34+ cells are able to efficiently counteract the ROS accumulation, as evidenced by the lower percentage of ROS positive cells at $24 \mathrm{~h}$ of treatment (Figure 1d).

Finally, to investigate whether this different level of oxidative stress entails a different ability to induce cell death, apoptosis was evaluated by means of Annexin V/PI staining. Our results show an increase of apoptosis level in CALR-mutated CD34+ cells compared to $J A K 2$-mutated ones, as shown in representative flow cytometry dot plots (Figure 1e).

These data further confirmed our previous results in CALR-mutated K562 cells [25], supporting a role for CALR mutation in ROS accumulation and in modulation of oxidative stress response in MF CD34+ primary cells. Moreover, our results showed that CALRmutated samples, where we observed a greater increase of intracellular ROS, have a higher apoptosis level compared to JAK2 ones.

\subsection{Modulation of SOD Activity in CD34+ Cells from JAK2- or CALR-Mutated MF Patients}

Among the response mechanisms to oxidative stress, SOD catalyzes the dismutation superoxide anions to hydrogen peroxide and oxygen, providing a defense against potential damage induced by ROS. To further study the cellular response to oxidative stress we assessed the SOD activity in wild-type and JAK2- or CALR-mutated CD34+ cells before and after $24 \mathrm{~h}$ Melittin treatment.

Our data show that the SOD activity is significantly decreased in MF patients' mutated cells compared to wild-type cells from HD in both Melittin treated and untreated condition (Figure 2a). Moreover, CALR-mutated cells show a greater decrease in SOD activity than $J A K 2$-mutated ones (Figure 2b). 
(a)

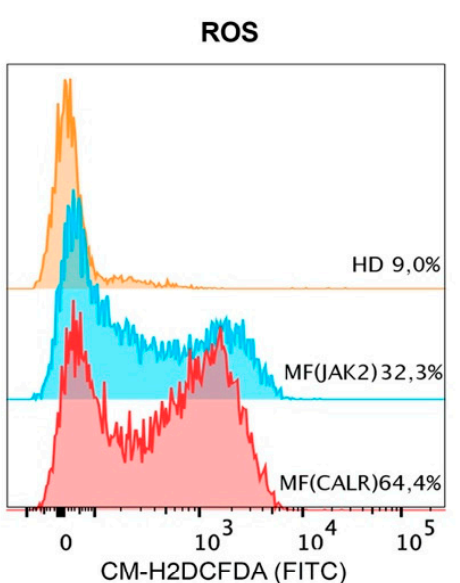

(d)

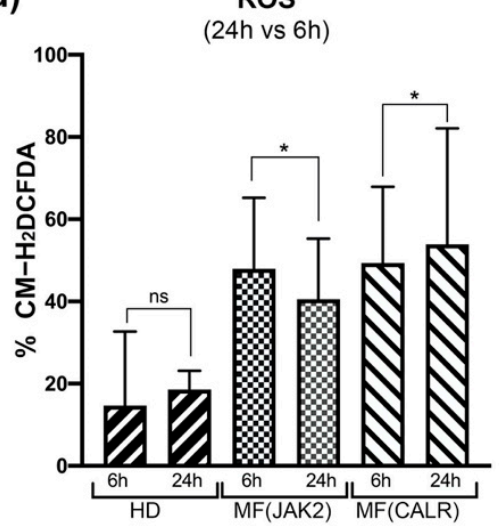

(b)

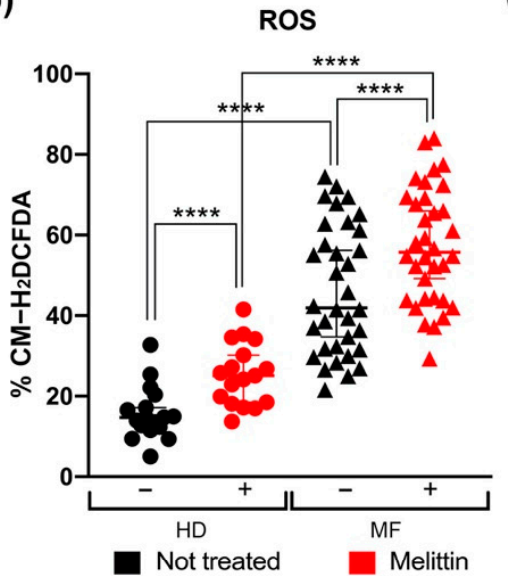

(e)

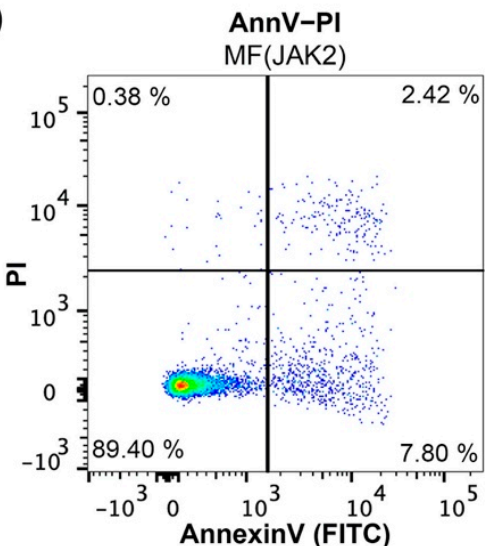

(c)
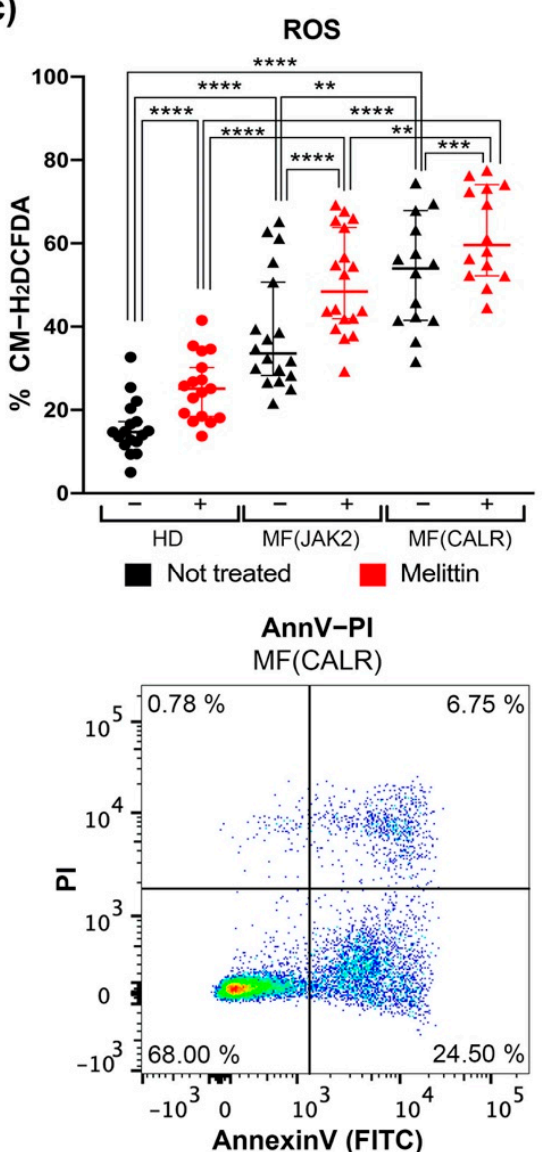

Figure 1. Detection of intracellular ROS levels in CD34+ cells of MF patients. (a) Representative histograms for flow cytometry detection of CM-H2DCFDA staining at $6 \mathrm{~h}$ in untreated CD34+ cells of $\mathrm{HD}, \mathrm{MF}(J A K 2)$ and $\mathrm{MF}(C A L R)$ samples. (b) Dot plot shows the percentages of intracellular ROS assessed in HD and in MF samples, with (red dots) or without (black dots) $6 \mathrm{~h}$ of Melittin treatment respectively. The comparison between HD and MF was analyzed using Mann-Whitney U test, while the comparisons between treated and untreated cells were analyzed with Wilcoxon matched-pairs signed rank test. (c) Dot plot shows the percentages of intracellular ROS in the comparison between $J A K 2$ - or CALR-mutated MF patients compared to HD, with (red dots) or without (black dots) $6 \mathrm{~h}$ Melittin treatment respectively. The comparisons between HD and MF(JAK2) and between HD and MF(CALR) was analyzed with Mann-Whitney U test, while the comparisons between treated and not treated cells were analyzed with Wilcoxon matched-pairs signed rank test. (d) Histogram shows the percentages of intracellular ROS after 6 and $24 \mathrm{~h}$; the statistical test used was Wilcoxon matched-pairs signed rank test. (e) Representative dot plots for flow cytometry detection of Annexin V/PI staining in CD34+ cells of $\operatorname{MF}(J A K 2)$ and $\operatorname{MF}(C A L R)$ samples. Data are reported as median with $95 \% \mathrm{CI}$ (confidence interval). ${ }^{*} p<0.05,{ }^{* *} p<0.01,{ }^{* * *} p<0.001,{ }^{* * * *} p<0.0001$.

\subsection{Quantification of Oxidative DNA Damage in CD34+ Cells from JAK2 and CALR-Mutated MF Patients}

In order to study the DNA damage secondary to ROS accumulation [9], we measured 8-OHdG level, as a biomarker of oxidative stress-mediated DNA damage [31].

Our results demonstrate that MF derived CD34+ cells have higher levels of 8-OHdG and this difference is even more evident after Melittin treatment, suggesting that, while cells from HDs are fully capable to respond to oxidative stress, mutated MF derived CD34+ cells display a greater sensitivity to oxidative DNA damage (Figure 3a). 
(a)

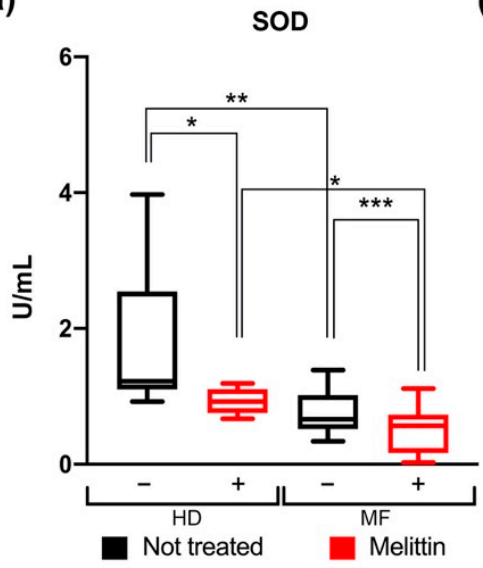

(b)

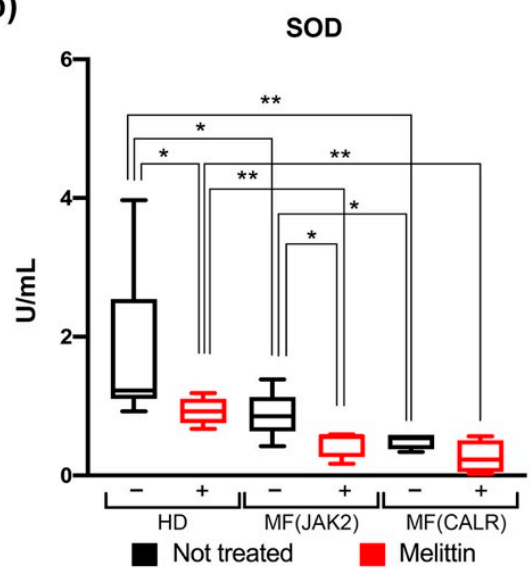

Figure 2. Measurement of SOD activity in CD34+ cells of MF patients. (a) Box plot shows the SOD activity in $\mathrm{HD}$ and in MF Scheme 24. hours Melittin treatment respectively. (b) Box plot shows the percentages of SOD activity in the comparison between JAK2 or CALR-mutated MF patients compared to HD, with (red bars) or without (black bars) $24 \mathrm{~h}$ Melittin treatment respectively. SOD activity was normalized based on total amount protein were used to perform the assay. Data are reported as median of SOD activity (expressed in $\mathrm{U} / \mathrm{mL}$ ) with $95 \% \mathrm{CI}$ (confidence interval). The comparisons between HD and MF were performed using Mann-Whitney $U$ test, while the comparisons between treated and not treated cells were analyzed using Wilcoxon matched-pairs signed rank test. ${ }^{*} p<0.05$, ${ }^{* *} p<0.01,{ }^{* * *} p<0.001$.

(a)

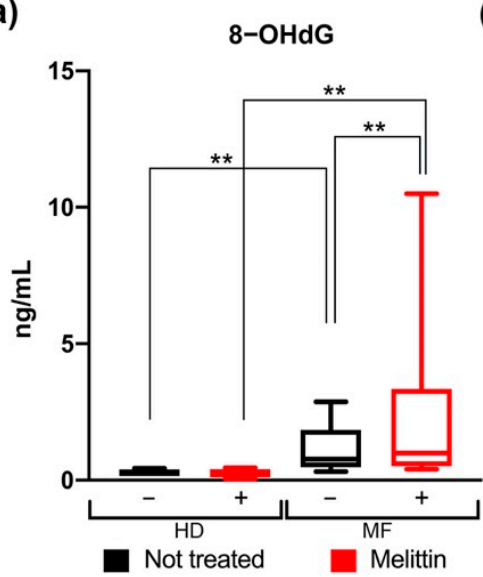

(b)

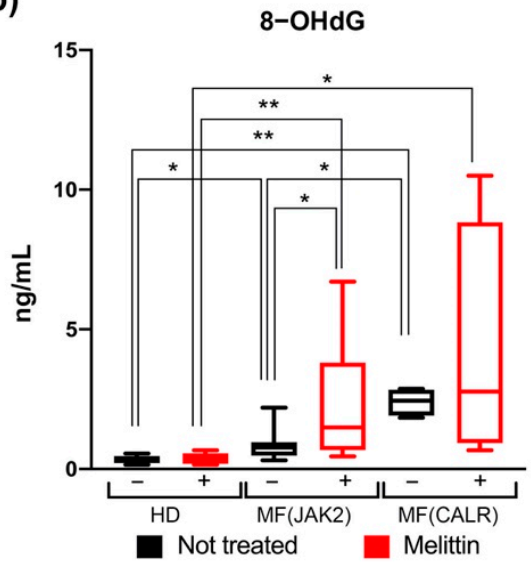

Figure 3. Measurement of 8-OHdG levels in CD34+ cells of MF patients. (a) Box plot shows the 8OHdG levels in HD and in MF samples, with (red bars) or without (black bars) $24 \mathrm{~h}$ Melittin treatment respectively. (b) Box plot shows the levels of 8-OHdG in JAK2- or CALR-mutated MF patients compared to HD, with (red bars) or without (black bars) $24 \mathrm{~h}$ of Melittin treatment respectively. Data are reported as median of $8-\mathrm{OHdG}$ levels (expressed in $\mathrm{ng} / \mathrm{mL}$ ) with $95 \% \mathrm{CI}$ (confidence interval). The comparisons between HD and MF were analyzed by means of Mann-Whitney U test, while the comparisons between treated and not treated cells were analyzed using Wilcoxon matched-pairs signed rank test. ${ }^{*} p<0.05,{ }^{* *} p<0.01$.

Regarding the differences between JAK2- and CALR-mutated cells, once again our results suggest that the effect of oxidative stress on DNA damage is greater for CALRmutated cells. CALR-mutated cells display higher 8-OHdG levels compared with HDs but also JAK2-mutated ones (Figure $3 \mathrm{~b}$ ). 


\subsection{Increased Plasma Levels of TAC in MF Patients Correlate with Clinical Detrimental Features}

Based on the results obtained thus far on CD34+ cells showing increased DNA damage induced by enhanced ROS levels in MF patients. Suggesting that CALR-mutated cells appear to be more sensible to oxidative stress, it seemed appropriate to investigate whether, in MF patients with different driver mutations, we could identify a biomarker for the response to oxidative stress that can be used in a clinical setting.

TAC is defined as the moles of oxidants neutralized by one liter of body fluids, such as plasma [32-34]. TAC is a parameter suitable for routine use in order to investigate the oxidative stress in many pathological conditions; therefore, we evaluated the level of TAC in 129 plasma samples from MF patients harboring CALR or JAK2 mutations $(n=86 J A K 2$, $n=43$ CALR).

Figure 4a shows that CALR-mutated patients have significantly lower TAC level if compared to JAK2-mutated ones, suggesting that the CALR mutation somehow causes a reduced antioxidant response.

(a)

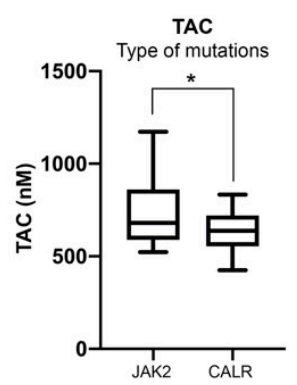

(e)

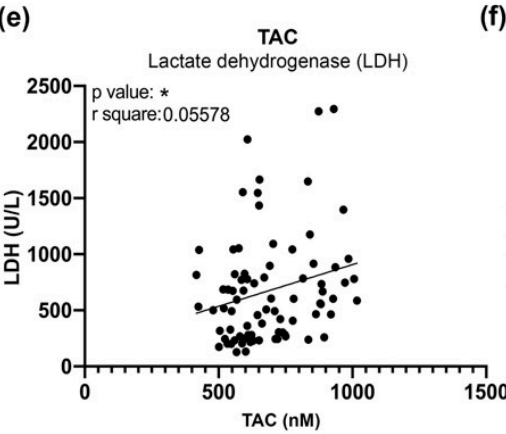

(b)

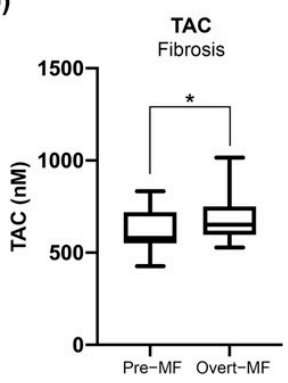

(f)

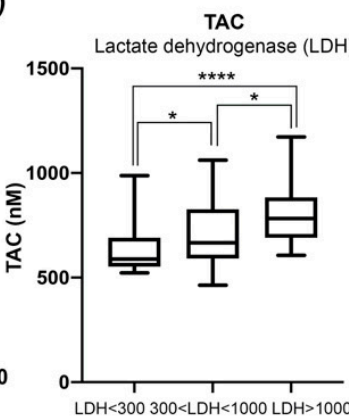

(c)

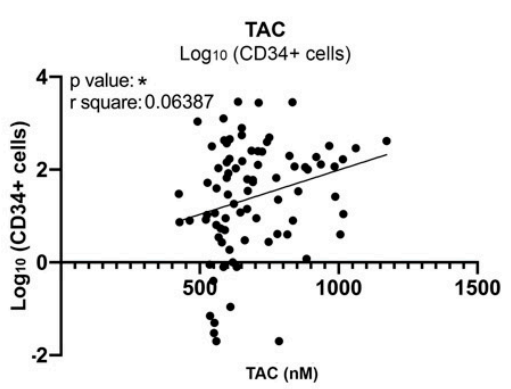

(g)

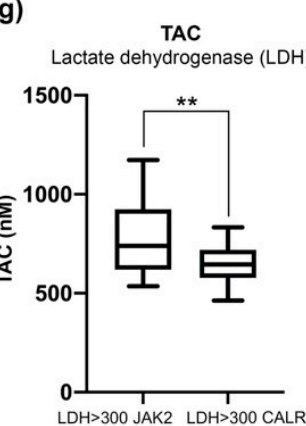

(d)

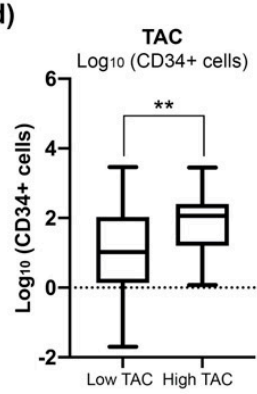

(h)

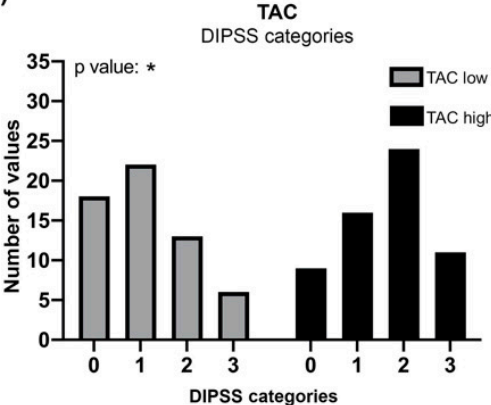

Figure 4. Measurement of TAC in plasma of MF patients and correlation with clinical detrimental features. (a) Box plot shows the level of TAC in CALR and JAK2-mutated MF patients. (b) Box plot shows correlation analysis of TAC level with fibrosis. (c) Graph represents linear regression analysis in MF patients showing correlation between the count of circulating CD34+ cells and TAC levels. (d) Box plot represents correlation analysis of the count of circulating CD34+ cells in MF samples presenting low or high levels of TAC. (e) Graph represents linear regression analysis in MF patients showing correlation between serum LDH and TAC levels. (f) Box plot represents correlation analysis of TAC levels with levels of serum LDH in MF samples divided into three ranges: $\mathrm{LDH}<300,300<\mathrm{LDH}$ $<1000$, LDH > $1000 \mathrm{nM}$. (g) Graph showing correlations between JAK2- and CALR-mutated patients with LDH > $300 \mathrm{nM}$ and TAC plasma levels. (h) Histogram was obtained from contingency tables computed to correlate low or high TAC plasma levels and DIPSS classification; the analysis was conducted with Chi-square test. Samples with low or high TAC levels are represented in gray and black, respectively. Box plot data are reported as median with $95 \% \mathrm{CI}$; the comparisons between two categories were analyzed with Mann-Whitney $\mathrm{U}$ test, while multiple comparisons were analyzed with Kruskal Wallis test. ${ }^{*} p<0.05,{ }^{* *} p<0.01,{ }^{* * *} p<0.0001$.

Next, in order to investigate the potential association between clinical features and TAC levels, we performed correlation analysis with a number of clinical parameters. Our 
results show that TAC activity significantly correlates with clinical parameters associated with MF severity, including the degree of fibrosis (Figure $4 \mathrm{~b}$ ), the numbers of circulating CD34+ cells (Figure 4c,d) and the levels of lactate dehydrogenase (LDH)(Figure 4e,f). Consistently with the data achieved thus far, among the patients with high levels of LDH, those who harbor CALR mutations display lower levels of TAC activity than those with $J A K 2$ mutations (Figure $4 \mathrm{~g}$ ).

Finally, we evaluated the correlation between TAC levels and patients' prognostic classification. To this end patients were classified according to Dynamic International Prognostic Scoring System (DIPSS) [35] and patients' cohort was stratified into two groups (Low and High) according to TAC plasma levels. The median value of TAC among MF samples $(642,189 \mathrm{nM})$ was used as cutoff according to the "median split" method.

As shown in Figure $4 \mathrm{~h}$, the frequency of High TAC samples is increased in DIPSS Intermediate-2 and High classes while Low TAC activity is more common in DIPSS Low and Intermediate- 1 categories. These data demonstrated that plasmatic TAC level is a detrimental feature that correlates with DIPSS classification.

\subsection{JAK2-Mutant MF Patients with High Level of TAC Are Associated with a Poor OS}

Given the described correlation between plasma TAC levels and detrimental clinical features we evaluated whether this parameter could be used as a marker of the patient's ability to react to the oxidative injury and as an outcome predictor.

Kaplan-Meier curves show that high levels of TAC correlate with a shorter OS $(\mathrm{HR}=2.304, p=0.0061)$ (Figure 5a). More specifically, patients could also be stratified according to driver mutation. When considering JAK2-mutated samples, high TAC levels identify a group of patients characterized by inferior OS, the same is true for CALRmutated samples where this difference approaches the statistical significance (Figure $5 \mathrm{~b}$ and Figure S2, Table 1).

Table 1. Individual comparisons between the four curves shown in Figure 5b. Kaplan-Meier estimates of OS according to TAC plasma levels and type of mutations (Figure S2 shows individual extended data). Differences between two survival curves was evaluated by log-rank (Mantel-Cox) test. Hazard ratio was computed to determine the magnitude of difference between two curves. $95 \%$ CI, $95 \%$ confidence interval. Significant log-rank $p$ values $(p<0.05)$ are represented in bold.

\begin{tabular}{ccc}
\hline \multirow{2}{*}{ Comparison } & \multicolumn{2}{c}{ Overall Survival } \\
\cline { 2 - 3 } & Hazard Ratio (95\% CI) & $p$ \\
\hline Low TAC-JAK2 vs. High TAC-JAK2 & $2.487(1.218-5.079)$ & $\mathbf{0 . 0 0 6 8}$ \\
\hline Low TAC-CALR vs. High TAC-CALR & $5.240(1.562-17.58)$ & 0.0694 \\
\hline Low TAC-JAK2 vs. Low TAC-CALR & $0.2756(0.07882-0.9635)$ & 0.1758 \\
\hline High TAC-JAK2 vs. High TAC-CALR & $0.6290(0.3073-1.288)$ & 0.2132 \\
\hline Low TAC-JAK2 vs. High TAC-CALR & $1.440(0.6341-3.271)$ & 0.3563 \\
\hline Low TAC-CALR vs. High TAC-JAK2 & $9.265(3.642-23.57)$ & $\mathbf{0 . 0 0 7 6}$ \\
\hline
\end{tabular}

Of particular interest, patients with $C A L R$ mutation and the lowest TAC activity are associated with better prognosis, while those with $J A K 2$ mutation and the highest levels of TAC activity are associated with worse outcome, as shown overall in Figure $5 \mathrm{~b}$ and then by individual comparisons between the groups in Table 1.

Moreover, we performed a multivariate cox regression analysis demonstrating that High TAC classification represents a risk factor for inferior survival when considering both driver mutation and the other detrimental features (Table 2). 
(a)

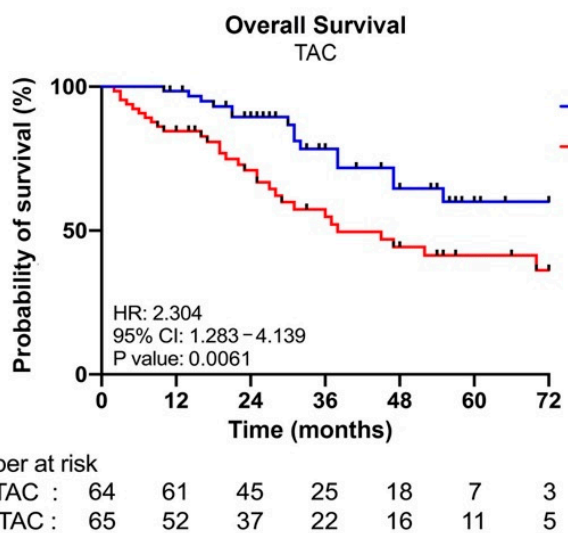

(c)

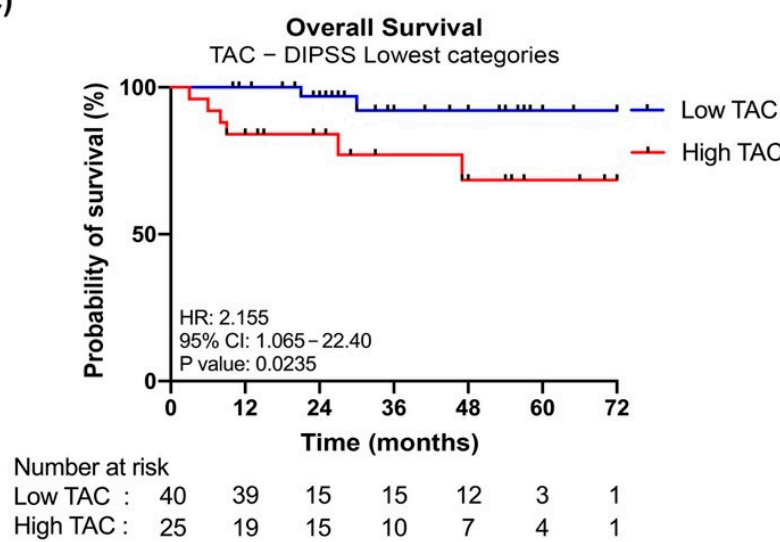

(b)

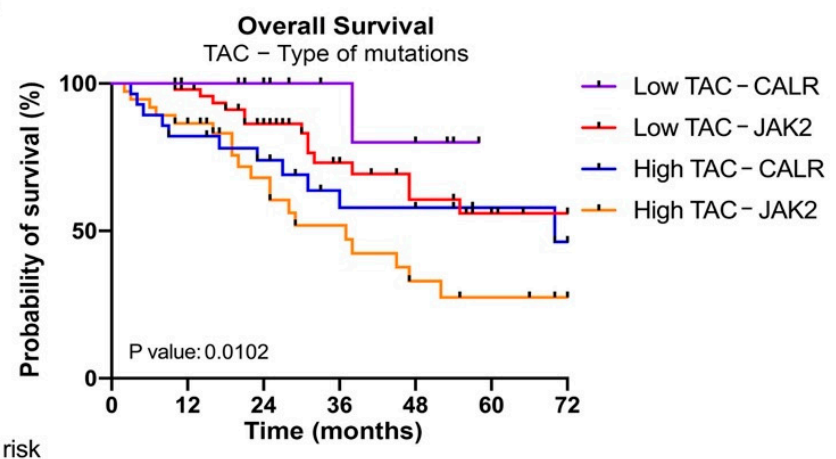

Number at risk

$\begin{array}{llllllll}\text { Low TAC -JAK2 } & : 49 & 47 & 34 & 20 & 16 & 7 & 3\end{array}$

Low TAC-CALR : $15 \quad 14 \quad 11 \quad 6 \quad 5011$

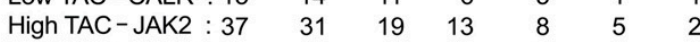

High TAC-CALR : $28 \quad 24 \quad 19 \quad 11 \quad 10 \quad 9 \quad 3$

(d)

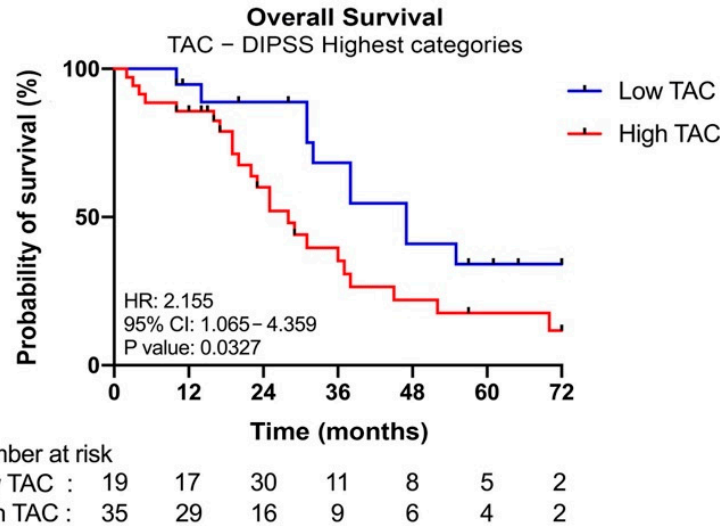

Figure 5. Kaplan-Meier analysis of OS according to TAC plasma levels. (a) Kaplan-Meier estimates OS according to TAC plasma levels. Patients' cohort was stratified into two groups (low and high) according to the plasma levels of TAC. Blue and red curves represent patients with low or high levels of TAC respectively. (b) Kaplan-Meier estimates of OS according to TAC plasma levels and type of mutations. For this analysis patient cohort was stratified into four groups: $C A L R$-mutated samples with low TAC (in violet), JAK2-mutated samples with low TAC (in red), CALR-mutated samples with high TAC (in blue), JAK2-mutated samples with high TAC (in yellow). (c) Kaplan-Meier estimates of OS according to TAC plasma levels in DIPSS lowest categories (Low and Intermediated-1). (d) Kaplan-Meier estimates of OS according to TAC plasma levels in DIPSS highest categories (High and Intermediated-2). Differences between two survival curves was evaluated by log-rank (MantelCox) test. HR, hazard ratio computed to determine the magnitude of differences between two curves. $95 \%$ CI, $95 \%$ confidence interval. $p$ value was computed by log-rank test.

Table 2. Results of multivariate regression analysis for overall survival. The prognostic impact of High TAC levels was determined while considering other detrimental features of MF patients by means of Cox regression analysis. Significant Wald test $p$ values $(p<0.05)$ are represented in bold. 95\% CI, 95\% confidence interval.

\begin{tabular}{ccc}
\hline \multirow{2}{*}{ Clinical Features } & \multicolumn{2}{c}{ Overall Survival } \\
\cline { 2 - 3 } & Hazard Ratio (95\% CI) & $p$ \\
\hline Presence of JAK2V17F & $3.8620(1.32-11.30)$ & $\mathbf{0 . 0 1 3 6 5}$ \\
\hline Overt MF & $0.5023(0.19-1.36)$ & 0.17510 \\
\hline Circulating CD34+ cells (Log10) & $1.7490(1.05-2.90)$ & $\mathbf{0 . 0 3 0 6 3}$ \\
\hline LDH > 1000 nM & $2.2210(0.77-6.39)$ & 0.13910 \\
\hline High TAC classification & $2.8910(1.18-7.1)$ & $\mathbf{0 . 0 0 6 8 3 8}$ \\
\hline
\end{tabular}


Finally, we performed survival analysis in samples dichotomized according to DIPSS to evaluate the independent prognostic value of TAC activity in MF. Patients with increased TAC activity display inferior survival when considering both DIPSS lowest (Low and Intermediate-1) and highest (Intermediate-2 and High) risk categories (Figure 5c,d) and multivariate analysis confirms that belonging to High TAC group is a risk factor for inferior survival independent from DIPSS classification (Hazard Ratio $=2.55 ; \mathrm{CI}=1.30-5.08$; $p=0.006838)$.

\section{Discussion}

MF is a clonal stem cell disorder characterized by dysregulated proliferation of myeloid cells, extramedullary hematopoiesis and excessive production of pro-inflammatory cytokines, resulting in chronic inflammation and genomic instability [5,36]. About $90 \%$ of $\mathrm{MF}$ patients carry JAK2, CALR or MPL mutations, which are often mutually exclusive and are referred to as "driver" mutations [5,37].

In particular, JAK2 is the most frequently mutated gene in MF and its mutation induces cytokine independence and constitutive activation of STAT proteins [13,38,39]. Moreover, it has been shown that JAK2 mutation induces the accumulation of ROS in the hematopoietic stem cell compartment of knock-in (KI) mouse model and in myelofibrosis patients, suggesting the importance of oxidative stress in MF pathogenesis [16].

In 2013, somatic CALR mutations were identified in the majority of JAK2 and MPL unmutated PMF and ET patients $[17,18]$. It has been shown that CALR-mutants binds to the Thrombopoietin receptor (TPO-R), causing its dimerization and leading again to the constitutive activation of JAK-STAT signaling pathway. Moreover, CALR overexpression has been shown to increase cell sensitivity to $\mathrm{H}_{2} \mathrm{O}_{2}$-induced cytotoxicity [24], indicating that CALR is also involved in the oxidative stress-induced apoptosis.

In the last decades, growing evidence has highlighted the role of oxidative stress in many tumors. Oxidative stress is considered as an imbalance between pro- and antioxidant species [1]. High levels of ROS are known to be involved in the pathogenesis of both solid and hematological cancers [40] and, in particular, a role in the initiation and progression of myeloproliferative disorders has been already described [2,3]. Here, we show that $J A K 2$ and CALR mutations impact differently on the oxidative stress in CD34+ cells from MF patients.

Our results demonstrate that MF CD34+ cells accumulate more ROS than those of healthy individuals, and ROS accumulation is associated with reduced SOD activity and increased DNA damage. These data show for the first time that the CALR mutation, more than JAK2 one, is a condition favoring the increase of both oxidative stress and DNA damage-related genomic instability. Moreover, our results show that CALR-mutated CD34+ cells, which exhibit greater increase of intracellular ROS, have a higher apoptosis level compared to JAK2-mutated ones, suggesting that mutated-CALR patients could activate protective mechanisms promoting cell death, while a moderate increase of ROS induces a lower level of cell death and could permit the survival of cancer cells allowing disease progression.

Our previous data on the dysregulation of the oxidative response in K652 cells overexpressing mutated $C A L R$ support the hypothesis that the increase in oxidative stress that we have demonstrated here in CALR-mutated myelofibrosis stem cells depends precisely on a molecular pathway mediated by mutated $C A L R$ rather than on other disease related mechanisms [25]. However, further studies should be necessary to elucidate the mechanism by which CALR mutation is able to induce oxidative stress. In this regard, we previously demonstrated in CALR-mutated K562 cells the downregulation of OXR1, a sensor that plays a critical role in protecting the cell against oxidative stress [23,25]. Moreover, we can suppose that alterations in calcium levels induced by CALR mutations might affect its ability to interact with transcription factors regulating the response to oxidative stress [24]. We could speculate that $C A L R$ mutations might impact on the protein ability to interact with transcription factors regulating the response to oxidative stress. 
As mentioned before, the final damage related to oxidative stress depends on the balance between the mechanisms supporting the production of ROS and the counterbalancing defense pathways. To further study this balance, we evaluated whether the differences in the level of oxidative stress between JAK2 and CALR-mutated patients correspond to a different ability in reacting to this insult. The assessment of the TAC has already been widely used as an index of the cellular ability to respond to oxidative injury [41].

In our patients' cohort, TAC levels significantly correlate with detrimental features such as the fibrosis grade, the peripheral CD34+ cells' number and with the inflammatory response measured by LDH levels. Our data show that plasma samples from patients with MF harboring CALR mutations show lower TAC levels than patients harboring JAK2 mutation.

In addition, since LDH is a critical enzyme of the anaerobic metabolic pathway, we also investigated the levels of L-Lactate in the plasma of MF patients. Cellular metabolism is strongly altered in pathological contexts and metabolic abnormalities is a hallmark of cancer. Lactate is produced by glycolysis, a major metabolic pathway responsible for glucose homeostasis and energy production [42]. Once considered merely a byproduct of glycolysis, lactate is now considered an important regulatory molecule of intermediate metabolism involved in cancer development and other diseases [43]. As shown in Figure S3, our results show an increase of L-Lactate in JAK2-mutated samples compared to CALR ones, suggesting that patients with the $J A K 2$ mutation have an increased anaerobic metabolism.

As a whole, these data suggest that $C A L R$-mutated patients are not only exposed to greater oxidative injury, but also show a reduced capacity to respond to damage. Conversely, JAK2-mutated patients show higher TAC, LDH and L-Lactate levels than CALR mutated ones.

Therefore, finally, we evaluated the association between TAC plasma levels and OS. Survival analysis performed on the High and Low TAC stratified cohort, shows that low TAC values correlate with better outcome. A further stratification of patients also considering the driver mutation allowed us to observe that the levels of TAC are however the strongest variable in determining the outcome. Patients with highest plasmatic TAC levels show inferior survival within both JAK2-mutated and CALR-mutated cohorts, and patients with highest TAC levels harboring JAK2 mutation display the worst prognosis, while patients with CALR mutations and low TAC levels show the best outcome. Moreover, multivariate analysis demonstrated that high TAC classification represents a prognostic factor for inferior survival independent from the type of driver mutation and the presence of other detrimental clinical features. The adverse prognostic impact of increased TAC activity on survival was also demonstrated by its correlation with DIPSS classification. Our results demonstrate that DIPSS highest categories are enriched in patients with high TAC levels, but we also observed that increased TAC is an independent prognostic factor allowing the identification of patients with inferior survival in both DIPSS lowest and highest categories.

In our working hypothesis, the biological interpretation of these results is to be sought in the balance between oxidative damage and response. A narrow concentration threshold determines whether ROS could act as second messengers, activating important cellular pathways, or induce cellular toxicity [44]. Moreover, ROS levels can be variable within cells, leading to an heterogeneity of induced damage [45]. Therefore, the type and degree of cellular response is crucial to determine the extent and intensity of ROS injury. High levels of ROS are able to induce oxidative stress, activating different cellular mechanisms which can lead to cell death. Conversely, a limited and moderate increase of ROS could permit the survival of DNA damaged cells [46]. As demonstrated by our results, JAK2-mutated CD34+ cells have lower levels of apoptosis and this might allow the survival of cancer cells, leading to the acquisition of new mutations that drive clonal evolution and disease progression, thus promoting the transition to the so-called "mutator-phenotype" described for MPNs [2,3]. 
This mechanistic hypothesis is also compatible with the already widely known evidence that patients with the CALR mutation generally have a better prognosis than patients with $J A K 2$-mutation [47], and adds the evidence that the assessment of response to oxidative damage, showed here using an easy plasma assay, could provide an additional variable to better define the prognosis of patients with myelofibrosis.

\section{Conclusions}

In summary, our results confirmed that $C A L R$ mutation has a higher impact than $J A K 2$ mutation on the oxidative stress status in MF cells. Furthermore, CALR-mutated plasma samples have significantly lower TAC levels leading to a lower responsiveness to oxidative injury.

Increased TAC levels correlate with the presence of $J A K 2$ mutation and several detrimental clinical features. MF patients with high plasmatic TAC display inferior survival and multivariate analysis demonstrated that increased TAC activity might represent a novel prognostic biomarker independent from DIPSS classification and other detrimental features.

We speculated that the high increase in oxidative stress in mutated CALR patients could be involved in the activation of protective mechanisms which ultimately promote cell death, while in patients with the JAK2 mutation, the slight increase in oxidative stress can be a mechanism that determines the persistence of cells with damaged DNA in which the accumulation of mutations promotes the disease progression.

Supplementary Materials: The following are available online at https: / www.mdpi.com/article / 10.3390/antiox11010113/s1, Figure S1: Gating strategy for intracellular ROS detection by flow cytometry; Figure S2: Kaplan-Meier analysis of OS according to TAC plasma levels and type of mutations; Figure S3: Measurement of L-Lactate in MF patients' plasma samples.

Author Contributions: Conceptualization, E.G. and R.M.; Data curation, E.G., M.M. (Margherita Mirabile), S.S., S.F., S.R., L.T., M.M. (Monica Maccaferri), P.G., E.B. and S.P.; Formal analysis, E.G., M.M. (Margherita Mirabile), S.S., S.F., S.R., L.T., E.B. and S.P.; Funding acquisition, R.M.; Investigation, E.G. and M.M. (Margherita Mirabile); Methodology, E.G. and M.M. (Margherita Mirabile); Project administration, R.M.; Resources, M.M. (Monica Maccaferri), P.G., C.C. (Corrado Colasante), R.P., T.O., B.M., L.P., F.P., M.T.V., E.T., M.L., A.M.V. and R.M.; Software, S.S., S.F., S.R., C.C. (Chiara Carretta), S.C. and E.T.; Supervision, E.G., A.M.V. and R.M.; Validation, S.S., S.F., S.R., L.T., M.M. (Monica Maccaferri), P.G., S.M., M.B., N.B., F.P., M.T.V., E.T., M.L. and A.M.V.; Visualization, E.G. and M.M. (Margherita Mirabile); Writing-original draft, E.G.; Writing-review and editing, M.M. (Margherita Mirabile) E.T., M.L., A.M.V. and R.M. All authors have read and agreed to the published version of the manuscript.

Funding: This research was funded by Associazione Italiana per la Ricerca sul Cancro: grant numbers 21267 and 19818; Ministero della Salute: grant number RF-2016-02362930; Ministero dell'Istruzione, dell'Università e della Ricerca: grant number 2017WXR7ZT_005.

Institutional Review Board Statement: The study was conducted according to the guidelines of the Declaration of Helsinki and approved by the Ethics Committee of Florence (Clearance of Ethical Commitee for Human experimentation of Florence: Comitato Etico Area Vasta dell'Azienda OspedalieroUniversitaria Careggi (approval file number \#2011/0014777, approval date: 22 April 2011).

Informed Consent Statement: Informed consent was obtained from all subjects involved in the study.

Data Availability Statement: Data are contained within the article or Supplementary Materials.

Acknowledgments: The complete membership of MYeloid NEoplasms Research Venture, AIRC (Mynerva) can be found at https:/ / progettomynerva.it/ (accessed on 1 December 2021).

Conflicts of Interest: The authors declare no conflict of interest. 


\section{References}

1. Sies, H. Biochemistry of Oxidative Stress. Angew. Chem. Int. Ed. Engl. 1986, 25, 1058-1071. [CrossRef]

2. Bjørn, M.E.; Hasselbalch, H.C. The Role of Reactive Oxygen Species in Myelofibrosis and Related Neoplasms. Mediat. Inflamm. 2015, 2015, 1-11. [CrossRef]

3. Allegra, A.; Pioggia, G.; Tonacci, A.; Casciaro, M.; Musolino, C.; Gangemi, S. Synergic Crosstalk between Inflammation, Oxidative Stress, and Genomic Alterations in BCR-ABL-Negative Myeloproliferative Neoplasm. Antioxidants 2020, 9, 1037. [CrossRef]

4. Tefferi, A.; Pardanani, A. Myeloproliferative Neoplasms: A Contemporary Review. JAMA Oncol. 2015, 1, 97. [CrossRef]

5. Tefferi, A. Primary Myelofibrosis: 2021 Update on Diagnosis, Risk-stratification and Management. Am. J. Hematol. 2021, 96, 145-162. [CrossRef] [PubMed]

6. On behalf of the International Working Group for Myelofibrosis Research and Treatment (IWG-MRT); Barosi, G.; Mesa, R.A.; Thiele, J.; Cervantes, F.; Campbell, P.J.; Verstovsek, S.; Dupriez, B.; Levine, R.L.; Passamonti, F.; et al. Proposed Criteria for the Diagnosis of Post-Polycythemia Vera and Post-Essential Thrombocythemia Myelofibrosis: A Consensus Statement from the International Working Group for Myelofibrosis Research and Treatment. Leukemia 2008, 22, 437-438. [CrossRef]

7. Vainchenker, W.; Kralovics, R. Genetic Basis and Molecular Pathophysiology of Classical Myeloproliferative Neoplasms. Blood 2017, 129, 667-679. [CrossRef] [PubMed]

8. Tefferi, A.; Vaidya, R.; Caramazza, D.; Finke, C.; Lasho, T.; Pardanani, A. Circulating Interleukin (IL)-8, IL-2R, IL-12, and IL-15 Levels Are Independently Prognostic in Primary Myelofibrosis: A Comprehensive Cytokine Profiling Study. JCO 2011, 29, 1356-1363. [CrossRef] [PubMed]

9. Yahata, T.; Takanashi, T.; Muguruma, Y.; Ibrahim, A.A.; Matsuzawa, H.; Uno, T.; Sheng, Y.; Onizuka, M.; Ito, M.; Kato, S.; et al. Accumulation of Oxidative DNA Damage Restricts the Self-Renewal Capacity of Human Hematopoietic Stem Cells. Blood 2011, 118, 2941-2950. [CrossRef]

10. Gloire, G.; Legrand-Poels, S.; Piette, J. NF-KB Activation by Reactive Oxygen Species: Fifteen Years Later. Biochem. Pharmacol. 2006, 72, 1493-1505. [CrossRef] [PubMed]

11. Vener, C.; Novembrino, C.; Bamonti Catena, F.; Fracchiolla, N.S.; Gianelli, U.; Savi, F.; Radaelli, F.; Fermo, E.; Cortelezzi, A.; Lonati, S. Oxidative Stress Is Increased in Primary and Post-polycythemia Vera Myelofibrosis. Exp. Hematol. 2010, 38, 1058-1065. [CrossRef] [PubMed]

12. Levine, R.L.; Wadleigh, M.; Cools, J.; Ebert, B.L.; Wernig, G.; Huntly, B.J.P.; Boggon, T.J.; Wlodarska, I.; Clark, J.J.; Moore, S.; et al. Activating Mutation in the Tyrosine Kinase JAK2 in Polycythemia Vera, Essential Thrombocythemia, and Myeloid Metaplasia with Myelofibrosis. Cancer Cell 2005, 7, 387-397. [CrossRef] [PubMed]

13. Kralovics, R.; Passamonti, F.; Buser, A.S.; Teo, S.-S.; Tiedt, R.; Passweg, J.R.; Tichelli, A.; Cazzola, M.; Skoda, R.C. A Gain-ofFunction Mutation of JAK2 in Myeloproliferative Disorders. N. Engl. J. Med. 2005, 352, 1779-1790. [CrossRef] [PubMed]

14. Hurtado-Nedelec, M.; Csillag-Grange, M.-J.; Boussetta, T.; Belambri, S.A.; Fay, M.; Cassinat, B.; Gougerot-Pocidalo, M.-A.; Dang, P.M.-C.; El-Benna, J. Increased Reactive Oxygen Species Production and P47phox Phosphorylation in Neutrophils from Myeloproliferative Disorders Patients with JAK2 (V617F) Mutation. Haematologica 2013, 98, 1517-1524. [CrossRef]

15. Djikic, D.; Markovic, D.; Bogdanovic, A.; Mitrovic-Ajtic, O.; Suboticki, T.; Diklic, M.; Beleslin-Cokic, B.; Bjelica, S.; Kovacic, M.; P Cokic, V. Oxidative and Nitrosative Stress in Myeloproliferative Neoplasms: The Impact on the AKT/MTOR Signaling Pathway. J. BUON 2018, 23, 1481-1491.

16. Marty, C.; Lacout, C.; Droin, N.; Le Couédic, J.-P.; Ribrag, V.; Solary, E.; Vainchenker, W.; Villeval, J.-L.; Plo, I. A Role for Reactive Oxygen Species in JAK2V617F Myeloproliferative Neoplasm Progression. Leukemia 2013, 27, 2187-2195. [CrossRef]

17. Nangalia, J.; Massie, C.E.; Baxter, E.J.; Nice, F.L.; Gundem, G.; Wedge, D.C.; Avezov, E.; Li, J.; Kollmann, K.; Kent, D.G.; et al. Somatic CALR Mutations in Myeloproliferative Neoplasms with Nonmutated JAK2. N. Engl. J. Med. 2013, 369, $2391-2405$. [CrossRef]

18. Klampfl, T.; Gisslinger, H.; Harutyunyan, A.S.; Nivarthi, H.; Rumi, E.; Milosevic, J.D.; Them, N.C.C.; Berg, T.; Gisslinger, B.; Pietra, D.; et al. Somatic Mutations of Calreticulin in Myeloproliferative Neoplasms. N. Engl. J. Med. 2013, 369, 2379-2390. [CrossRef]

19. Michalak, M.; Corbett, E.F.; Mesaeli, N.; Nakamura, K.; Opas, M. Calreticulin: One Protein, One Gene, Many Functions. Biochem. J. 1999, 344 Pt 2, 281-292. [CrossRef]

20. Elf, S.; Abdelfattah, N.S.; Chen, E.; Perales-Paton, J.; Rosen, E.A.; Ko, A.; Peisker, F.; Florescu, N.; Giannini, S.; Wolach, O.; et al. Mutant Calreticulin Requires Both Its Mutant C-Terminus and the Thrombopoietin Receptor for Oncogenic Transformation. Cancer Discov. 2016, 6, 368-381. [CrossRef]

21. Araki, M.; Yang, Y.; Masubuchi, N.; Hironaka, Y.; Takei, H.; Morishita, S.; Mizukami, Y.; Kan, S.; Shirane, S.; Edahiro, Y.; et al. Activation of the Thrombopoietin Receptor by Mutant Calreticulin in CALR-Mutant Myeloproliferative Neoplasms. Blood 2016, 127, 1307-1316. [CrossRef]

22. Araki, M.; Yang, Y.; Imai, M.; Mizukami, Y.; Kihara, Y.; Sunami, Y.; Masubuchi, N.; Edahiro, Y.; Hironaka, Y.; Osaga, S.; et al. Homomultimerization of Mutant Calreticulin Is a Prerequisite for MPL Binding and Activation. Leukemia 2019, 33, 122-131. [CrossRef]

23. Zhang, Y.; Liu, L.; Jin, L.; Yi, X.; Dang, E.; Yang, Y.; Li, C.; Gao, T. Oxidative Stress-Induced Calreticulin Expression and Translocation: New Insights into the Destruction of Melanocytes. J. Investig. Dermatol. 2014, 134, 183-191. [CrossRef] [PubMed] 
24. Ihara, Y.; Urata, Y.; Goto, S.; Kondo, T. Role of Calreticulin in the Sensitivity of Myocardiac H9c2 Cells to Oxidative Stress Caused by Hydrogen Peroxide. Am. J. Physiol. Cell Physiol. 2006, 290, C208-C221. [CrossRef] [PubMed]

25. Salati, S.; Genovese, E.; Carretta, C.; Zini, R.; Bartalucci, N.; Prudente, Z.; Pennucci, V.; Ruberti, S.; Rossi, C.; Rontauroli, S.; et al Calreticulin Ins5 and Del52 Mutations Impair Unfolded Protein and Oxidative Stress Responses in K562 Cells Expressing CALR Mutants. Sci. Rep. 2019, 9, 10558. [CrossRef]

26. Arber, D.A.; Orazi, A.; Hasserjian, R.; Thiele, J.; Borowitz, M.J.; Le Beau, M.M.; Bloomfield, C.D.; Cazzola, M.; Vardiman, J.W. The 2016 Revision to the World Health Organization Classification of Myeloid Neoplasms and Acute Leukemia. Blood 2016, 127, 2391-2405. [CrossRef] [PubMed]

27. Fantini, S.; Rontauroli, S.; Sartini, S.; Mirabile, M.; Bianchi, E.; Badii, F.; Maccaferri, M.; Guglielmelli, P.; Ottone, T.; Palmieri, R.; et al. Increased Plasma Levels of LncRNAs LINC01268, GAS5 and MALAT1 Correlate with Negative Prognostic Factors in Myelofibrosis. Cancers 2021, 13, 4744. [CrossRef] [PubMed]

28. Shah, J.S.; Soon, P.S.; Marsh, D.J. Comparison of Methodologies to Detect Low Levels of Hemolysis in Serum for Accurate Assessment of Serum MicroRNAs. PLoS ONE 2016, 11, e0153200. [CrossRef]

29. Bianchi, E.; Ruberti, S.; Rontauroli, S.; Guglielmelli, P.; Salati, S.; Rossi, C.; Zini, R.; Tagliafico, E.; Vannucchi, A.; Manfredini, R. Role of MiR-34a-5p in Hematopoietic Progenitor Cells Proliferation and Fate Decision: Novel Insights into the Pathogenesis of Primary Myelofibrosis. IJMS 2017, 18, 145. [CrossRef]

30. Gajski, G.; Domijan, A.-M.; Žegura, B.; Štern, A.; Gerić, M.; Novak Jovanović, I.; Vrhovac, I.; Madunić, J.; Breljak, D.; Filipič, M.; et al. Melittin Induced Cytogenetic Damage, Oxidative Stress and Changes in Gene Expression in Human Peripheral Blood Lymphocytes. Toxicon 2016, 110, 56-67. [CrossRef]

31. Shibutani, S.; Takeshita, M.; Grollman, A.P. Insertion of Specific Bases during DNA Synthesis Past the Oxidation-Damaged Base 8-OxodG. Nature 1991, 349, 431-434. [CrossRef]

32. Serafini, M.; Villano, D.; Spera, G.; Pellegrini, N. Redox Molecules and Cancer Prevention: The Importance of Understanding the Role of the Antioxidant Network. Nutr. Cancer 2006, 56, 232-240. [CrossRef]

33. Lettieri-Barbato, D.; Tomei, F.; Sancini, A.; Morabito, G.; Serafini, M. Effect of Plant Foods and Beverages on Plasma NonEnzymatic Antioxidant Capacity in Human Subjects: A Meta-Analysis. Br. J. Nutr. 2013, 109, 1544-1556. [CrossRef]

34. Peluso, I.; Cavaliere, A.; Palmery, M. Plasma Total Antioxidant Capacity and Peroxidation Biomarkers in Psoriasis. J. Biomed. Sci. 2016, 23, 52. [CrossRef] [PubMed]

35. Passamonti, F.; Cervantes, F.; Vannucchi, A.M.; Morra, E.; Rumi, E.; Pereira, A.; Guglielmelli, P.; Pungolino, E.; Caramella, M.; Maffioli, M.; et al. A Dynamic Prognostic Model to Predict Survival in Primary Myelofibrosis: A Study by the IWG-MRT (International Working Group for Myeloproliferative Neoplasms Research and Treatment). Blood 2010, 115, 1703-1708. [CrossRef]

36. Tefferi, A. Myelofibrosis with Myeloid Metaplasia. N. Engl. J. Med. 2000, 342, 1255-1265. [CrossRef] [PubMed]

37. Pardanani, A.D.; Levine, R.L.; Lasho, T.; Pikman, Y.; Mesa, R.A.; Wadleigh, M.; Steensma, D.P.; Elliott, M.A.; Wolanskyj, A.P.; Hogan, W.J.; et al. MPL515 Mutations in Myeloproliferative and Other Myeloid Disorders: A Study of 1182 Patients. Blood 2006, 108, 3472-3476. [CrossRef] [PubMed]

38. James, C.; Ugo, V.; Le Couédic, J.-P.; Staerk, J.; Delhommeau, F.; Lacout, C.; Garçon, L.; Raslova, H.; Berger, R.; Bennaceur-Griscelli, A.; et al. A Unique Clonal JAK2 Mutation Leading to Constitutive Signalling Causes Polycythaemia Vera. Nature 2005, 434, 1144-1148. [CrossRef]

39. Levine, R.L.; Pardanani, A.; Tefferi, A.; Gilliland, D.G. Role of JAK2 in the Pathogenesis and Therapy of Myeloproliferative Disorders. Nat. Rev. Cancer 2007, 7, 673-683. [CrossRef]

40. Waris, G.; Ahsan, H. Reactive Oxygen Species: Role in the Development of Cancer and Various Chronic Conditions. J. Carcinog. 2006, 5, 14. [CrossRef] [PubMed]

41. Bartosz, G. Total Antioxidant Capacity. In Advances in Clinical Chemistry; Elsevier: Amsterdam, The Netherlands, 2003; Volume 37, pp. 219-292. ISBN 978-0-12-010337-9.

42. Philp, A.; Macdonald, A.L.; Watt, P.W. Lactate-A Signal Coordinating Cell and Systemic Function. J. Exp. Biol. 2005, 208, 4561-4575. [CrossRef] [PubMed]

43. Brooks, G.A. The Science and Translation of Lactate Shuttle Theory. Cell Metab. 2018, 27, 757-785. [CrossRef]

44. Lushchak, V.I. Free Radicals, Reactive Oxygen Species, Oxidative Stress and Its Classification. Chem. Biol. Interact. 2014, 224, 164-175. [CrossRef]

45. Testa, U.; Labbaye, C.; Castelli, G.; Pelosi, E. Oxidative Stress and Hypoxia in Normal and Leukemic Stem Cells. Exp. Hematol. 2016, 44, 540-560. [CrossRef] [PubMed]

46. Sabharwal, S.S.; Schumacker, P.T. Mitochondrial ROS in Cancer: Initiators, Amplifiers or an Achilles' Heel? Nat. Rev. Cancer 2014, 14, 709-721. [CrossRef]

47. Beer, P.A.; Campbell, P.J.; Green, A.R. Comparison of Different Criteria for the Diagnosis of Primary Myelofibrosis Reveals Limited Clinical Utility for Measurement of Serum Lactate Dehydrogenase. Haematologica 2010, 95, 1960-1963. [CrossRef] [PubMed] 